\title{
PRETTY RATIONAL MODELS FOR POINCARÉ DUALITY PAIRS
}

\author{
HECTOR CORDOVA BULENS, PASCAL LAMBRECHTS, \\ AND DON STANLEY
}

\begin{abstract}
We prove that a large class of Poincaré duality pairs admit rational models (in the sense of Sullivan) of a particularly nice form associated to some Poincaré duality CDGA. These models have applications in particular to the construction of rational models of configuration spaces in compact manifolds with boundary.
\end{abstract}

\section{INTRODUCTION}

Sullivan theory [9] encodes the rational homotopy type of a simplyconnected space of finite type, $X$, into a commutative differential graded algebra $(\mathrm{CDGA}),\left(A, d_{A}\right)$, such that $\mathrm{H}\left(A, d_{A}\right) \cong \mathrm{H}^{*}(X ; \mathbb{Q})$ and which is called a $C D G A$ model of $X$ (see Section 2.1 fo a quick recapitulation on that theory). In [7] we proved that when $X$ is a simply-connected Poincaré duality space (the most important example being a closed manifold), we can always construct a CDGA model whose underlying algebra satisfies Poincaré duality. These Poincaré duality CDGA models are often convenient and were used for example in [5] and in [8] to construct nice rational models for configuration spaces in closed manifolds, or in [4] to study the Chas-Sullivan product on the free loop space.

The aim of this paper is to exhibit convenient CDGA models for Poincaré duality pairs of spaces, like compact manifolds with boundary. Such a model should be a CDGA morphism between two CDGAs representing each element of the pair. Our main result is that a very large class of Poincaré duality pairs admit what we call pretty models (Definition 3.1.) More precisely we will show that the following Poincaré duality pairs admit such models:

- even dimensional disk bundles over a simply-connected closed manifold relative to their sphere bundles (Theorem 4.1);

Date: 18 May 2015.

2010 Mathematics Subject Classification. 55P62, 55M05.

Key words and phrases. Poincaré duality, Sullivan model, Commutative differential graded algebra.

H.C.B. and P.L. are supported by the belgian federal fund PAI Dygest. 
- Poincaré duality pairs $(W, \partial W)$ where $\partial W$ is 2-connected and retracts rationally on its half-skeleton (Definition 6.1 and Theorem 6.6);

- the complement of a subpolyhedron of high codimension in a closed manifold (relative to its natural boundary) (end of Section 3 and Theorem 6.6 applied to Exemple 6.4 (2) .)

Let us describe roughly the form of these pretty models (see Section 3 for more details). A pretty model for $\partial W \hookrightarrow W$ is a CDGA morphism between mapping cones.

$$
\varphi \oplus \mathrm{id}: P \oplus_{\varphi} ! s s^{-n} \# Q \longrightarrow Q \oplus_{\varphi \varphi}: s s^{-n} \# Q
$$

where

- $P$ is a Poincaré duality CDGA in dimension $n=\operatorname{dim} W$ (see Definition 5.1);

- $\varphi: P \rightarrow Q$ is a CDGA morphism;

- $s s^{-n} \# Q$ is the $(n-1)$ - th suspension of the linear dual $\# Q=$ $\operatorname{hom}_{\mathbb{Q}}(Q, \mathbb{Q}) ;$

- $\varphi^{!}: s^{-n} \# Q \rightarrow P$ is a $P$-dgmodule morphism constructed out of $\varphi$ and the Poincaré duality isomorphism on $P$;

- the CDGA structure on the mapping cones is the semi-trivial one described at Section 2.2 (which requires that $\varphi \varphi^{!}$is balanced in the sense of Definition 2.1.)

In the special case when $\partial W=\emptyset$, we have $Q=0$ and we recover a Poincaré duality CDGA model, $P$, for $W$ as in [7]

Note also that the model

$$
Q \oplus_{\varphi \varphi} s s^{-n} \# Q
$$

is a Poincaré duality CDGA model in dimension $n-1$ for $\partial W$.

When $\partial W \neq \emptyset, W$ is not a Poincaré duality space and thus does not admit a Poincaré duality CDGA model. However, often $W$ has a model which is an explicit quotient of a Poincaré duality space, as shows the following

Proposition 1.1 (Corollary [3.3). If $(W, \partial W)$ admits a pretty model (11) and if $\varphi$ is surjective, then $W$ has a CDGA model

$$
P / I
$$

where $P$ is a Poincaré duality $C D G A$ and $I=\varphi^{!}\left(s^{-n} \# Q\right)$ is a differential ideal.

These pretty models should be very convenient in many constructions in rational homotopy theory on Poincaré duality pairs. In particular we use them in [2] and [1] to obtain explicit models for the configuration spaces in a manifold with boundary.

Here is the plan of the paper. In Section 2 we review quickly basic facts and terminology about rational homotopy theory, and we define 
the semi-trivial CDGA structure on some mapping cones. In Section 3 we define pretty models for Poincaré duality pairs and we motivate this definition by the example of the complement of a polyhedron in a closed manifold. In Section 4 we prove that even dimensional disk bundles over simply-connected Poincaré duality spaces admit pretty models. Section 5 is devoted to the construction of nice Poincaré duality CDGA modelling a given CDGA whose cohomology satisfies Poincaré duality; these results will be needed in the next section. We prove in Section 6 that any Poincaré duality pair whose boundary is 2-connected and retracts rationally on its half skeletton admits a pretty model. In the last section we wonder whether every Poincaré duality pair admits a pretty model.

\section{CDGA's, DGmodules, AND SEMi-Trivial CDGA structures ON MAPPING CONES}

2.1. Rational homotopy theory. In this paper we will use the standard tools and results of rational homotopy theory, following the notation and terminology of [3]. Recall that $A_{P L}$ is the Sullivan-de Rham contravariant functor and that for a 1-connected space of finite type, $X, A_{P L}(X)$ is a commutative differential graded algebra (CDGA for short, always non-negatively graded.) Any CDGA weakly equivalent to $A_{P L}(X)$ is called a $C D G A$ model of $X$ and it completely encodes the rational homotopy type of $X$. Similarly a CDGA model of a map of spaces $X \rightarrow Y$ is a CDGA morphism weakly equivalent to $A_{P L}(f): A_{P L}(Y) \rightarrow A_{P L}(X)$. All our dgmodules and CDGAs are over the field $\mathbb{Q}$. A CDGA, $A$, is connected if $A^{0}=\mathbb{Q}$. A Poincaré $d u$ ality $C D G A$ is a connected CDGA whose underlying algebra satisfies Poincaré duality (see Definition 5.1 for a precise definition.)

2.2. Mapping cones and semi-trivial CDGA structures. Let $A$ be a CDGA and let $R$ be an $A$-dgmodule. We will denote by $s^{k} R$ the kth suspension of $R$, i.e. $\left(s^{k} R\right)^{p}=R^{k+p}$, and for a map of $A$-dgmodules, $f: R \rightarrow Q$, we denote by $s^{k} f$ the k-th suspension of $f$. For example, $s^{-n} \mathbb{Q}$ is a dgmodule concentrated in degree $n$. Furthermore, we will use \# to denote the linear dual of a vector space, $\# V=\operatorname{hom}(V, \mathbb{Q})$, and \#f to denote the linear dual of a map $f$. A dgmodule is of finite type if it is of finite dimension in every degree. If $M$ is a dgmodule, we write $M^{>k}=0$ to express that $M^{i}=0$ for each $i>k$; similarly we will write $M^{\geq k}=0, M^{<k}=0$, etc.

If $f: Q \rightarrow R$ is an $A$-dgmodule morphism, the mapping cone of $f$ is the $A$-dgmodule

$$
C(f):=(R \oplus s Q, \delta)
$$


defined by $R \oplus s Q$ as an $A$-module and with a differential $\delta$ such that $\delta(r, s q)=\left(d_{R}(r)+f(q),-s d_{Q}(q)\right)$. We also write $C(f)=R \oplus_{f} s Q$. When $f=0$, we just write $C(0)=R \oplus s Q$.

When $R=A$, the mapping cone $C(f: Q \rightarrow A)$ can be equipped with a unique commutative graded algebra (CGA) structure that extends the algebra structure on $A$ and the $A$-dgmodule structure on $s Q$, and such that $(s q) \cdot\left(s q^{\prime}\right)=0$, for $q, q^{\prime} \in Q$. We will call this structure the semi-trivial structure on the mapping cone $A \oplus_{f} s Q$ (see [6, Section 4]).

Definition 2.1. Let $A$ be a CDGA. An A-dgmodule morphism $f: Q \rightarrow$ $A$ is balanced if, for each $x, y \in Q$ :

$$
f(x) \cdot y=x \cdot f(y) .
$$

The importance of this notion comes from the following proposition:

Proposition 2.2. Let $Q$ be an A-dgmodule and $f: Q \rightarrow A$ be an $A$ dgmodule morphism. If $f$ is balanced then the mapping cone $C(f)=$ $A \oplus_{f} s Q$ endowed with the semi-trivial CGA structure is a CDGA.

Proof. The only non trivially verified condition for $C(f)$ being a CDGA is the Leibniz rule for the differential. Let $a, a^{\prime} \in A$ and $q, q^{\prime} \in Q$. For products of the form $(a, 0) \cdot\left(a^{\prime}, 0\right)$ and of the form $(a, 0) \cdot(0, s q)$ the Leibniz rule is verified because $A$ is a CDGA and $Q$ is an $A$-dgmodule. For products of the form $(0, s q) \cdot\left(0, s q^{\prime}\right)$, by semi-trivality of the CDGA structure of the mapping cone we have to verify that

$$
(\delta(0, s q)) \cdot\left(0, s q^{\prime}\right)+(-1)^{|q|+1}(0, s q) \cdot\left(\delta\left(0, s q^{\prime}\right)\right)=0,
$$

which is a consequence of the hypothesis that $f$ is balanced.

Remark 2.3. In the rest of this paper, when a mapping cone is equipped with a CDGA structure it will be understood that is comes from the semi-trivial structure.

\section{Pretty models}

In this section we first describe precisely what we call pretty models, and next we motivate this definition by showing that these models arise naturally as models of complements of a subpolyhedron in a closed manifold.

Suppose given

(i) a connected Poincaré duality CDGA, $P$, in dimension $n$ (see Definition 5.1 below);

(ii) a connected CDGA, $Q$;

(iii) a CDGA morphism, $\varphi: P \rightarrow Q$.

By definition of a Poincaré duality CDGA, there exists an isomorphism of $P$-dgmodules

$$
\theta_{P}: P \stackrel{\cong}{\longrightarrow} s^{-n} \# P
$$


which is unique up to multiplication by a non zero scalar because $P$ is a free $P$-module generated by 1 .

Consider the composite

$$
\varphi^{!}: s^{-n} \# Q \stackrel{s^{-n} \# \varphi}{\longrightarrow} s^{-n} \# P \stackrel{\theta_{P}^{-1}}{\longrightarrow} P,
$$

which is a morphism of $P$-dgmodule. It is a shriek map or top degree map in the sense of [6], because $\mathrm{H}^{n}\left(\varphi^{!}\right)$is an isomorphism.

Assume that

$$
\varphi \varphi^{!}: s^{-n} \# Q \rightarrow Q
$$

is balanced (see Definition 2.1). Then $\varphi^{!}$is also balanced because the $P$-module structure on $s^{-n} \# Q$ is induced throught $\varphi$. By Proposition 2.2 the mapping cones

$$
P \oplus_{\varphi} ! s s^{-n} \# Q \quad \text { and } \quad Q \oplus_{\varphi \varphi} s s^{-n} \# Q
$$

are CDGA and

$$
\varphi \oplus i d: P \oplus_{\varphi} ! s s^{-n} \# Q \longrightarrow Q \oplus_{\varphi \varphi} ! s s^{-n} \# Q
$$

is a CDGA morphism.

Definition 3.1. Let $\varphi: P \longrightarrow Q$ be a CDGA morphism with $P$ a connected Poincaré duality CDGA in dimension $n$, consider $\varphi^{!}$: $s^{-n} \# Q \longrightarrow P$ defined at (4), and assume that $\varphi \varphi^{!}$is balanced. Then the CDGA morphism

$$
\varphi \oplus i d: P \oplus_{\varphi} ! s s^{-n} \# Q \longrightarrow Q \oplus_{\varphi \varphi}: s s^{-n} \# Q
$$

is called the pretty model associated to $\varphi$. If moreover $\varphi$ is surjective, we say that it is a surjective pretty model.

Proposition 3.2. If (6) is a surjective pretty model then the projection

$$
\pi: P \oplus_{\varphi} ! s s^{-n} \# Q \stackrel{\simeq}{\longrightarrow} P / I,
$$

where $I:=\varphi^{!}\left(s^{-n} \# Q\right)$, is a quasi-isomorphism of CDGA.

Proof. $I=\varphi^{!}\left(s^{-n} \# Q\right)$ is a differential ideal of $P$ because it is the image of a morphism of $P$-dgmodules. Since $\varphi$ is surjective, by duality, $\varphi^{!}$is injective and we have a short exact sequence

$$
0 \longrightarrow s^{-n} \# Q \stackrel{\varphi^{!}}{\longrightarrow} P \stackrel{\operatorname{proj}}{\longrightarrow} P / I \longrightarrow 0 .
$$

Thus

$$
\pi:=\left(\operatorname{proj}_{0} 0\right): P \oplus_{\varphi}: s s^{-n} \# Q \longrightarrow P / I
$$

is a quasi-isomorphism of CDGA.

Corollary 3.3. If a Poincaré duality pair $(W, \partial W)$ admits a surjective pretty model (6), then a CDGA model of $W$ is given by $P / I$ where $P$ is a Poincaré duality $C D G A$. 
To motivate the above definition, let us show how pretty models appear naturally as models of the complement of a subpolyhedron in a closed manifold. Let $M$ be a simply-connected closed triangulated manifold and let $K$ be a subpolyhedron and assume that $\operatorname{dim}(M) \geq$ $2 \operatorname{dim}(K)+3$. The complement $M \backslash K$ is a open manifold which is the interior of a compact manifold $W$ whose boundary, $\partial W$, is the boundary of a thickening of $K$ in $M$. Let

$$
\varphi: P \rightarrow Q
$$

be a CDGA model of the inclusion $K \hookrightarrow M$ where $P$ is a Poincaré duality CDGA model of $M$ and $Q^{\geq n / 2-1}=0$ (by Proposition 5.4, such a model exists under our high codimension hypothesis.) Consider the morphism $\varphi^{!}$defined as the composite (4) which is a shriek map in the sense of [6, Definition 5.1]. By the main result of that paper, [6, Theorem 1.2], the pretty model (5) is then a CDGA model of the inclusion $\partial W \hookrightarrow W$ (using the fact that $\varphi \varphi^{!}=0$, and hence is balanced, for degree reasons.) This example show that pretty models appear naturally as models of complements of a subpolyhedron of high codimension.

Notice also that any compact manifold with boundary, $W$, arises as the complement of a subpolyhedron in a closed manifold. Indeed we can consider the double $M:=W \cup_{\partial} W^{\prime}$, where $W^{\prime}$ is a second copy of $W$. Then $M$ is a closed manifold and $W$ is the complement of $W^{\prime}$ in $M$. Of course, $W^{\prime}$ is not necessarily of high homotopical codimension, thus the discussion above does not apply stricly to this example, which explains why this construction does not directly imply that any compact manifold with boundary admit a pretty model.

Note also that when $W$ is the complement of a high codimension subpolyhedron in a closed manifold, then the boundary, $\partial W$, retracts rationally on its half skeletton (in the sense of Definition 6.1, see Example 6.4), and therefore Theorem 6.6 gives another proof that this complement admits a pretty model.

\section{Disk BUndles over Poincaré DUALity SPACES}

In this section we prove that we can construct explicit pretty models for the total space of an even-dimensional disk bundle over a closed manifold.

Theorem 4.1. Let $\xi$ be a vector bundle of even rank over a simplyconnected Poincaré duality space. Then the pair $(D \xi, S \xi)$ of associated (disk, sphere) bundles admits a surjective pretty model.

Moreover this model can be explicitly constructed out of any CDGA model of the base and from the Euler class of the bundle.

Proof. Assume that $\xi$ is of rank $2 k$ and that the base is a Poincaré duality space in dimension $n-2 k$. Recall from [7] or Definition 5.1 the notion of a Poincaré duality CDGA. Let $Q$ be a Poincaré duality 
CDGA model of the base (which, by [7] or Proposition [5.5, exists and can be explictely constructed out of any CDGA model of the base) and let $e \in Q^{2 k} \cap$ ker $d$ be a representative of the Euler class of the bundle. Then a CDGA model of the sphere bundle is given by

$$
Q \longmapsto(Q \otimes \wedge z, d z=e)
$$

with $\operatorname{deg}(z)=2 k-1$, and this is also a model of the pair $(D \xi, S \xi)$.

Denote by $\bar{z}$ a generator of degree $2 k$ and define the CDGA

$$
P:=\left(\frac{Q \otimes \wedge \bar{z}}{\left(\bar{z}^{2}-e \bar{z}\right)}, D \bar{z}=0\right)
$$

which is a Poincaré duality CDGA in dimension $n$ (where $\left(\bar{z}^{2}-e \bar{z}\right)$ is the ideal in $Q \otimes \wedge \bar{z}$ generated by this difference). As vector spaces we have $P \cong Q \oplus \bar{z} \cdot Q$. Define the CDGA morphism

$$
\varphi: P \longrightarrow Q
$$

by $\varphi\left(q_{1}+q_{2} \bar{z}\right)=q_{1}+e \cdot q_{2}$, for $q_{1}, q_{2} \in Q$.

We will show that the pretty model associated to $\varphi$ is equivalent to the CDGA morphism (7), which will establish the proposition. Consider the following diagram

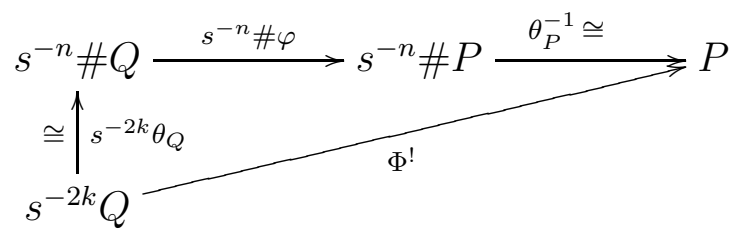

where $\theta_{Q}$ and $\theta_{P}$ are the Poincaré duality isomorphisms, and we set $\varphi^{!}:=\theta_{P}^{-1} \circ\left(s^{-n} \# \varphi\right)$ and $\Phi^{!}:=\varphi^{!} \circ\left(s^{-2 k} \theta_{Q}\right)$. We prove that we can assume that $\Phi^{\text {! }}$ is given by

$$
\Phi^{!}\left(s^{-2 k} q\right)=q \cdot \bar{z} .
$$

Indeed $\Phi^{!}\left(s^{-2 k} 1\right)=\alpha+\lambda \bar{z}$ for some $\alpha \in Q^{2 k}$ and $\lambda \in Q^{0}=\mathbb{Q}$. Since $\Phi^{!}$is a morphism of $P$-dgmodules, $\bar{z} \cdot \Phi^{!}\left(s^{-2 k} 1\right)=\Phi^{!}\left(\bar{z} \cdot s^{-2 k} 1\right)$ which implies, using that $\bar{z} \cdot s^{-2 k} 1=e \cdot s^{-2 k} 1$,

$$
\alpha \bar{z}+\lambda \bar{z}^{2}=e \alpha+\lambda e \bar{z}
$$

and therefore, since $\bar{z}^{2}=e \bar{z}, \alpha=0$. Also $\lambda \neq 0$ because $\Phi^{!}$induces an isomorphism in $\mathrm{H}^{n}(-)$, since $s^{-n} \# \varphi$ does. We can replace the isomorphism $\theta_{P}$ by $\lambda \cdot \theta_{P}$ and we get

$$
\Phi^{!}\left(s^{-2 k} 1\right)=\bar{z}
$$

which implies (8).

A direct computation shows that $\varphi \Phi^{!}$is balanced, and hence also $\varphi \varphi$ !. The pretty model associated to $\varphi$ is isomorphic to

$$
\varphi \oplus \mathrm{id}: P \oplus_{\Phi} ! s s^{-2 k} Q \longrightarrow Q \oplus_{\varphi \Phi^{!}} s s^{-2 k} Q .
$$


The codomain of $\varphi \oplus$ id is isomorphic to $(Q \otimes \wedge z, d z=e)$ because $\varphi \Phi^{!}\left(s^{-2 k} 1\right)=e$. The inclusion of $Q$ in the domain of $\varphi \oplus \mathrm{id}$,

$$
Q \hookrightarrow P \hookrightarrow P \oplus_{\Phi !} s s^{-2 k} Q,
$$

is clearly a quasi-isomorphism. Thus $\varphi \oplus$ id is weakly equivalent to (7), which is a model of $(D \xi, S \xi)$, and the proposition is proved.

\section{Poincaré duality CDGA's}

In this section we recall that any 1-connected closed manifold admits a Poincaré duality CDGA-model, and we prove some relative version of that result (Proposition 5.4.) This will be used in the next section to build more pretty models.

Any 1-connected closed $n$-dimensional manifold, $M$, satisfies Poincaré duality in degree $n$, which means that there is an isomorphism

$$
\mathrm{H}^{*}(M ; \mathbb{Q}) \cong \# \mathrm{H}^{n-*}(M ; \mathbb{Q})
$$

of $\mathrm{H}^{*}(M ; \mathbb{Q})$-modules. In [7] we proved that Poincaré duality holds not only in cohomology but also on some CDGA model of $M$. To make this precise, we review the following

Definition 5.1. An oriented Poincaré duality CDGA in dimension $n$, or PDCDGA, is a connected CDGA of finite type, $P$, equipped with an isomorphism of $P$-dgmodules

$$
\theta_{P}: P \stackrel{\cong}{\rightarrow} s^{-n} \# P .
$$

Remark 5.2. Since $P$ is a free $P$-dgmodule generated by a single element, the isomorphism $\theta_{P}$ of (9) is unique up to a multiplication by a non-zero scalar. When this isomorphism is not specified, we talk of a Poincaré duality CDGA (dropping the adjective oriented.)

Remark 5.3. It is easy to check that Definition 5.1] is equivalent to [7, Definition 2.2]. Indeed the orientation $\epsilon: P \rightarrow s^{-n} \mathbb{Q}$ of [7, Definition 2.2-2.3] is obtained by $\epsilon:=\theta_{P}(1)$, where $1 \in \mathbb{Q}$. Conversely, the isomorphism $\theta_{P}$ is obtained from the orientation $\epsilon$ by $\left(\theta_{P}(y)\right)(x):=\epsilon(x . y)$.

The main result of [7] is that any CDGA whose cohomology is 1connected and satisfies Poincaré duality is weakly equivalent to some Poincaré duality CDGA. The aim of this section is to prove the following relative version of that result:

Proposition 5.4. Let $\psi: A \rightarrow B$ be a morphism of CDGA such that $\mathrm{H}(A)$ is a 1-connected Poincaré duality algebra in dimension $n$, $\mathrm{H}^{\geq \frac{n}{2}-1}(B)=0, \mathrm{H}(B)$ is 1-connected and of finite type, and $\mathrm{H}^{2}(\psi)$ is surjective.

Then $\psi$ is equivalent to some surjective CDGA morphism

$$
\varphi: P \rightarrow Q
$$


such that $P$ is a 1-connected Poincaré duality CDGA in dimension n, $Q$ is 1-connected, and $Q^{\geq \frac{n}{2}-1}=0$. Moreover the morphism $\varphi$ can be constructed explicitely out of the morphism $\psi$. Also, if $B$ is 1-connected and $B^{\geq \frac{n}{2}-1}=0$ then we can take $Q=B$.

A key ingredient to prove this proposition is the following:

Proposition 5.5. Let $A$ be a CDGA such that $\mathrm{H}(A)$ is a Poincaré duality algebra in dimension $n$. Assume moreover that $n \geq 7, A$ is 1-connected of finite type, and $A^{2} \subset \operatorname{ker} d$.

Then there exists a CDGA quasi-isomorphism

$$
\lambda: A \stackrel{\simeq}{\longrightarrow} P
$$

such that $P$ is a Poincaré duality CDGA in dimension $n$ and $\lambda$ is an isomorphism in degrees $<\frac{n}{2}-1$.

This proposition is an improvement of the main result of [7] in the sense that the quasi-isomorphism $\rho$ to the Poincare duality CDGA is an isomorphism below about half the dimension.

If we take for granted Proposition 5.5 then we can prove Proposition 5.4 as follows.

Proof of Proposition 5.4. If $n \leq 6$ then, since $\mathrm{H}^{\geq \frac{n}{2}-1}(B)=0$ and $\mathrm{H}(B)$ is 1-connected, we have $\mathrm{H}(B)=\mathbb{Q}$ and the proposition is a consequence of the main result of [7] by taking $Q=\mathbb{Q}$.

Assume now that $n \geq 7$. By passing to Sullivan models it is easy to see that $\psi$ is equivalent to a surjective morphism between 1-connected finite type CDGA's. Thus without loss of generality we assume that $\psi: A \rightarrow B$ is already like that. Moreover, since $\mathrm{H}^{\geq \frac{n}{2}-1}(B)=0$, by moding out $B$ by a suitable acyclic ideal we get a surjective quasiisomorphism

where $Q^{\geq \frac{n}{2}-1}=0$.

$$
\pi: B \stackrel{\simeq}{\rightarrow} Q
$$

By Proposition [5.5, there is a quasi-isomorphism $\lambda: A \stackrel{\simeq}{\rightarrow} P$ which is an isomorphism in degrees $<n / 2-1$ and such that $P$ is a Poincaré duality CDGA. Since $(\operatorname{ker} \lambda)^{<\frac{n}{2}-1}=0$ and $Q^{\geq \frac{n}{2}-1}=0$, the morphism $\pi \psi$ extends along $\lambda$ into the desired morphism $\varphi: P \rightarrow Q$.

The rest of this section is devoted to the proof of Proposition 5.5. Since the techniques used here will not appear in the rest of this paper, the reader can safely jump to the next section if he wishes. The proof is based on techniques of [7] and we assume that the reader is familiar with the notation and proofs of that paper. In particular, recall that an orientation (in degree $n$ ) of a $\operatorname{CDGA}\left(A, d_{A}\right)$ is a chain map

$$
\epsilon: A \rightarrow s^{-n} \mathbb{Q}
$$

that is surjective in cohomology [7, Definition 2.3]. We then say that $(A, d, \epsilon)$ is an oriented $C D G A$. Note that $\epsilon$ is a chain map if and only 
10HECTOR CORDOVA BULENS, PASCAL LAMBRECHTS, AND DON STANLEY

if $\epsilon\left(d\left(A^{n-1}\right)\right)=0$. The differential ideal of orphans of this oriented CDGA is ([7, Definition 3.1 and Proposition 3.2])

$$
\mathcal{O}=\mathcal{O}(A, \epsilon):=\{a \in A \text { such that } \forall b \in A: \epsilon(a \cdot b)=0\} .
$$

The main interest of the notion of orphans is that when $A$ is 1-connected of finite type and $\mathrm{H}(A)$ is a Poincaré duality algebra in degree $n$ then $P=A / \mathcal{O}$ is a Poincaré duality CDGA in degree $n$ [7, Proposition 3.3]. Moreover, $\mathrm{P}$ is quasi-isomorphic to $A$ when $\mathcal{O}$ is acyclic. The strategy of the proof of Proposition 5.5 is to build a Sullivan extension $(A, d) \longmapsto(\hat{A}:=A \otimes \wedge V, \hat{d})$ such that $V^{<\frac{n}{2}-1}=0$ and its ideal of orphans $\hat{\mathcal{O}}$ is acyclic and without elements of degree $<\frac{n}{2}-1$. Then $\lambda: A \longrightarrow \hat{A} / \hat{\mathcal{O}}$ will be the desired quasi-isomorphism to a PDCDGA. For the sake of the proof we need the following definition:

Definition 5.6. Let $(A, d, \epsilon)$ be an oriented $C D G A$.

(i) The oriented $C D G A(A, \epsilon)$ has no orphans in degrees $\leq p$ if $(\mathcal{O}(A, \epsilon))^{i}=0$ for $i \leq p$.

(ii) An acyclic oriented Sullivan extension is a Sullivan extension

$$
(A, d) \stackrel{\simeq}{\longrightarrow}(\hat{A}:=A \otimes \wedge V, \hat{d})
$$

that is a quasi-isomorphism and is equipped with an orientation $\hat{\epsilon}: \hat{A} \rightarrow s^{-n} \mathbb{Q}$ that extends $\epsilon: A \rightarrow s^{-n} \mathbb{Q}$.

(iii) The acyclic oriented Sullivan extension (ii) adds no orphans in degree $\leq q$ if

$$
(\mathcal{O}(\hat{A}, \hat{\epsilon}))^{i} \subset(\mathcal{O}(A, \epsilon))^{i} \quad \text { for } i \leq q .
$$

(iv) The acyclic oriented Sullivan extension (ii) adds no generators in degree $\leq m$ if

$$
V^{i}=0 \text { for } i \leq m \text {. }
$$

Proposition 5.5 will be a consequence of an inductive application of the following two lemmas.

Lemma 5.7. Let $(A, d, \epsilon)$ be an oriented $C D G A$ of finite type that is 1-connected, such that $A^{2} \subset \operatorname{ker}(d)$ and $\mathrm{H}(A, d)$ is a Poincaré duality algebra in degree $n \geq 7$.

Then $(A, d, \epsilon)$ admits an acyclic oriented Sullivan extension that adds no orphans in degree $\leq \frac{n}{2}-1$, adds no generators in degrees $<\frac{n}{2}-1$, and whose set of orphans is acyclic.

Proof. The set of orphans of $(A, d, \epsilon)$ is $\frac{n}{2}$-half-acyclic (see [7, Definition $3.5]$ and the remark after). Since, by hypothesis, $(A, d, \epsilon)$ satisfies [7, (4.1)] we can apply [7, Proposition 5.1] iteratively for all integers $k$ ranging from $\left\lceil\frac{n}{2}+1\right\rceil$ up to $n+1$. More precisely, at each step we construct the extension described at [7, Section 4] for the integer $k \geq$ 
$\frac{n}{2}+1$. This is an acyclic Sullivan extension defined at the equation [7, (4.4)] which is oriented by [7, Lemma 4.5]. The set of orphans in this extension is $k$-half-acyclic by [7, Proposition 5.1].

The new generators of lowest degrees in the extension [7, (4.4)] are the $w_{i}$ 's of degree $k-2 \geq \frac{n}{2}-1$. Thus the extension adds no generator of degrees $<\frac{n}{2}-1$.

Since by [7, (4.1)], $d \gamma_{i}=\alpha_{i}$ and since, by [7, (4.2)], $\alpha_{i}$ is not the boundary of an orphan in $A$, there exists $\xi_{i} \in A$ such that $\epsilon\left(\gamma_{i} \xi_{i}\right) \neq 0$. By [7, (4.5)(ii)], $\hat{\epsilon}\left(w_{i} d\left(\xi_{i}\right)\right)= \pm \epsilon\left(\gamma_{i} \xi_{i}\right) \neq 0$ and therefore $w_{i}$ is not an orphan in $\hat{A}$. Thus the extension adds no orphans in degrees $\leq k-2$, hence in degrees $\leq \frac{n}{2}-1$.

When we reach $k=n+1$, the set of orphans is $(n+1)$-half acyclic, and therefore is acyclic by [7, Proposition 3.6].

Lemma 5.8. Let $(A, d, \epsilon)$ be as in Lemma 5.7. Assume that its set of orphans is acyclic and that there are no orphans in degrees $<p$ for some integer $1 \leq p<\frac{n}{2}-1$. Then $(A, d, \epsilon)$ admits an acyclic oriented Sullivan extension with no orphans in degrees $\leq p$ and which adds no generators in degrees $\leq \frac{n}{2}$.

Proof. Let $\mathcal{O}$ be the ideal of orphans in $(A, d, \epsilon)$. Since $\mathcal{O}$ is acyclic and $\mathcal{O}^{<p}=0$, we have $\mathcal{O}^{p} \cap \operatorname{ker}(d)=0$. Let $\left\{x_{1}, \ldots, x_{r}\right\}$ be a basis of $\mathcal{O}^{p}$. Consider the acyclic Sullivan extension

$$
\hat{A}:=\left(A \otimes \wedge\left(u_{1}, \ldots, u_{r}, \bar{u}_{1}, \ldots, \bar{u}_{r}\right), \hat{d}\right)
$$

with $\operatorname{deg}\left(u_{i}\right)=n-p-1, \operatorname{deg}\left(\bar{u}_{i}\right)=n-p, \hat{d}\left(u_{i}\right)=\bar{u}_{i}$ and $\hat{d}\left(\bar{u}_{i}\right)=0$. We extend the orientation $\epsilon$ into an orientation $\hat{\epsilon}$ of $\hat{A}$ as follows. Let $S$ be a supplement space of $\mathcal{O}^{p} \oplus\left(A^{p} \cap \operatorname{ker}(d)\right)$ in $A^{p}$. Let $T$ be a supplement space of $d\left(\mathcal{O}^{p}\right) \oplus d(S)$ in $A^{p+1}$. Since $n-p-1>\frac{n}{2}$ there is a unique degree 0 map

$$
\hat{\epsilon}: \hat{A} \rightarrow s^{-n} \mathbb{Q}
$$

extending $\epsilon$ and such that

$$
\begin{cases}\hat{\epsilon}\left(\bar{u}_{i} \cdot x_{j}\right) & =\delta_{i j} \quad \text { where } \delta_{i j} \text { is the Kronecker symbol } \\ \hat{\epsilon}\left(\bar{u}_{i} \cdot \operatorname{ker}(d)\right) & =0 \\ \hat{\epsilon}\left(\bar{u}_{i} \cdot S\right) & =0 \\ \hat{\epsilon}\left(u_{i} \cdot d\left(x_{j}\right)\right) & =(-1)^{n-p} \delta_{i j} \\ \hat{\epsilon}\left(u_{i} \cdot d(S)\right) & =0 \\ \hat{\epsilon}\left(u_{i} \cdot T\right) & =0 .\end{cases}
$$

Then one computes that $\hat{\epsilon}\left(d\left(\hat{A}^{n-1}\right)\right)=0$, and hence $\hat{\epsilon}$ is an orientation.

This extension adds no generators in degrees $<n-p-1$, and hence no generators in degrees $\leq \frac{n}{2}$ (because $p<\frac{n}{2}-1$.) For the same reasons it adds no orphans in degrees $\leq p$. Moreover all the degree $p$ orphans of $A$, which are linear combinations of $x_{1}, \ldots, x_{r}$, are not 
orphans anymore in $\hat{A}$ since $\hat{\epsilon}\left(\bar{u}_{i} \cdot x_{j}\right)=\delta_{i j}$. Thus $\hat{A}$ has no orphans in degree $\leq p$.

Proof of Proposition 5.5. Let $p_{\max }$ be the largest integer $<\frac{n}{2}-1$. Apply Lemmas 5.7, 5.8, and5.7 again, successively for $p=1,2, \ldots, p_{\max }$. This gives by composition an acyclic oriented Sullivan extension $\hat{A}$ with an acyclic ideal of orphans, with no orphans in degrees $<\frac{n}{2}-1$, and with no generators added in degree $<\frac{n}{2}-1$. Therefore the composite

$$
\lambda: A \stackrel{\simeq}{\longrightarrow} \hat{A} \stackrel{\simeq}{\longrightarrow} \frac{\hat{A}}{\mathcal{O}(\hat{A}, \hat{\epsilon})}
$$

is a quasi-isomorphism, and an isomorphism in degrees $<\frac{n}{2}-1$, to a Poincaré duality CDGA.

\section{Poincaré Duality spaces that Retract Rationally on THEIR HALF-SKELETON}

In this section we consider a quite large class of Poincaré duality pairs $(W, \partial W)$ that admit a pretty model. Indeed the only restriction is on the boundary $\partial W$ which should retracts rationally on its half-skeleton as explained in the following definition.

Definition 6.1. Let $M$ be a simply-connected Poincaré duality space in dimension $n-1$. We say that it retracts rationally on its half-skeleton if there exists a morphism of connected CDGAs

$$
\rho: Q \longrightarrow A
$$

such that

(i) $A$ is a $C D G A$ model of $M$,

(ii) $\mathrm{H}^{\geq n / 2-1}(Q)=0$, and

(iii) $\mathrm{H}^{k}(\rho)$ is an isomorphism for $k \leq n / 2$.

Remark 6.2. The terminology comes from the fact that the conditions of the definition imply that the realization of $\rho$ can be thought of as a retraction of $M$ on a skeleton of half the dimension, as it is clear from Diagram (10) in the proof of Proposition [6.5.

Remark 6.3. Poincaré duality and (ii)-(iii) in the previous definition imply that $M$ has no cohomology about the middle dimension. More precisely, if $n$ is even then $\mathrm{H}^{n / 2-1}(M)=\mathrm{H}^{n / 2}(M)=0$, and if $n$ is odd then $\mathrm{H}^{(n-1) / 2}(M)=0$.

Exemple 6.4. (1) Consider the total space $W$ of a d-dimensional disk bundle over a closed manifold of dimension $<d-1$. Then $\partial W$ retracts rationally on its half-skeleton as one checks by building a model of the sphere bundle. 
(2) Let $K$ be a compact polyhedra embedded in a closed manifold $M$ of dimension $n$. Assume that $K$ and $M$ are 1-connected and $M \geq 2 \operatorname{dim} K+3$. Let $T$ be a regular neighborhood of $K$ in $M$. Then $N:=\partial T$ retracts rationally on its half-skeleton. Indeed, [6. Theorem 1.2] gives a model of $\partial T$ of the form $Q \oplus s D$ where $Q$ is a model of $K$ and $D \simeq s^{-n} \# Q$, and the conclusion follows.

(3) As a special case of the previous example consider a 1-connected polyhedron $K$ embedded in $S^{n}=\mathbb{R}^{n} \cup\{\infty\}$ with $n \geq 2 \operatorname{dim} K+3$. Then the boundary of a thickening of $K$ in $S^{n}$ retracts rationally on its half-skeleton.

Proposition 6.5. Let $M$ be a simply-connected Poincaré duality space in dimension $n-1$. Then it retracts rationally on its half-skeleton if and only if there exists a connected $C D G A, Q$, such that

(i) $Q^{\geq n / 2-1}=0$, and

(ii) $Q \oplus s s^{-n} \# Q$ is a $C D G A$ model of $M$.

Proof. It is clear that (i) and (ii) imply that $M$ retract rationally on its half-skeleton.

Let us prove the converse. Let

$$
\rho^{\prime}: Q^{\prime} \longrightarrow A^{\prime}
$$

be a morphism between connected CDGAs that satisfies (i)-(iii) of Definition 6.1 (with the added decoration "prime"). Consider a minimal Sullivan extension $\hat{\rho}$ :

$$
Q^{\prime} \stackrel{\hat{\rho}}{\longrightarrow}\left(Q^{\prime} \otimes \wedge V, D^{\prime}\right) \stackrel{\simeq}{\longrightarrow} A^{\prime}
$$

that factors $\rho^{\prime}$. Let $h$ be the integer such that $n=2 h$ or $n=2 h+1$. Since $\mathrm{H}^{\leq h}(\rho)$ is an isomorphism and $\mathrm{H}^{>h}\left(Q^{\prime}\right)=0$, by minimality we have $V^{\leq h}=0$. Since $Q^{\prime}$ is connected and $\mathrm{H}^{\geq n / 2-1}\left(Q^{\prime}\right)=0$, there exists an acyclic ideal $J \subset Q^{\prime}$ such that $Q^{\prime \geq n / 2-1} \subset J$. Set $Q:=Q^{\prime} / J$ and consider the push-out of CDGAs

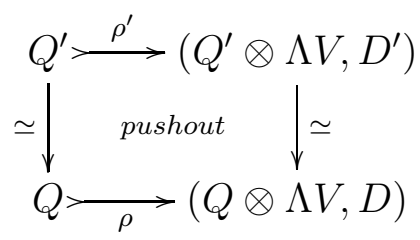

and set $A:=(Q \otimes \wedge V, D)$. Note that $\rho$ is an isomorphism in degrees $\leq h$. Also it endows $A$ with the structure of a $Q$-dgmodule.

Let $S$ be a supplement of $A^{h} \cap \operatorname{ker} d$ in $A^{h}$ and set

$$
I:=S \oplus A^{>h},
$$

which is an ideal since $A$ is connected. For degree reasons and since $\mathrm{H}^{\leq h}(\rho)$ is an isomorphism, the composite

$$
Q \stackrel{\rho}{\longrightarrow} A \stackrel{\text { proj }}{\longrightarrow} A / I
$$


is a quasi-isomorphism.

By the lifting lemma [3, Proposition 14.6], in the following diagram

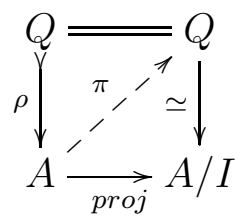

we get a CDGA morphism $\pi$ that makes the upper left triangle commute and the lower right triangle commute up to homotopy, in other words, $Q$ is a retract of $A$.

Since $\mathrm{H}(A)$ satisfies Poincaré duality in dimension $n-1$, there exists a quasi-isomorphism of $A$-dgmodules, hence of $Q$-dgmodules,

$$
\theta: A \stackrel{\simeq}{\longrightarrow} s s^{-n} \# A,
$$

and we have the diagram

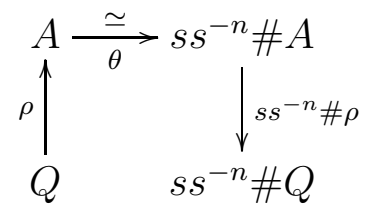

Set $\lambda=\left(s s^{-n} \# \rho\right) \circ \theta$ which is a morphism of $Q$-dgmodules. Since $\rho$ induces an isomorphism in homology in degrees $\leq n / 2$, we get that $s s^{-n} \# \rho$, and hence $\lambda$, induces an isomorphism in homology in degrees $\geq n / 2-1$.

Consider the $Q$-dgmodule morphism

$$
\epsilon=(\pi, \lambda): A \longrightarrow Q \oplus s s^{-n} \# Q .
$$

For degree reasons and since $\pi$ (respectively, $\lambda$ ) induces isomorphism in homology below (respectively, above) degree $n / 2$, we get that $\epsilon$ is a quasi-isomorphism.

We prove that $\epsilon$ is a morphism of algebras. Let $a, a^{\prime} \in A$. If $\operatorname{deg}(a) \leq$ $h$, then, since $\rho$ is an isomorphism in that degree, the multiplication by $a$ is determined by the $Q$-module structure, and since $\epsilon$ is of $Q$ module we get that $\epsilon\left(a \cdot a^{\prime}\right)=\epsilon(a) \cdot \epsilon\left(a^{\prime}\right)$. The same arguments work if $\operatorname{deg}\left(a^{\prime}\right) \leq h$. If both $a$ and $a^{\prime}$ are of degrees $\geq h+1$, then $\operatorname{deg}\left(a \cdot a^{\prime}\right)>n$ and then $\epsilon\left(a \cdot a^{\prime}\right)=0=\epsilon(a) \cdot \epsilon\left(a^{\prime}\right)$, for degree reasons.

Thus $\epsilon$ is a CDGA quasi-isomorphism and the proposition is proved.

Theorem 6.6. Let $W$ be a simply-connected compact manifold with boundary $\partial W$. Assume that $\partial W$ is 2-connected and retracts rationally on its half-skeleton. Then $(W, \partial W)$ admits a surjective pretty model.

Proof. Since $\partial W$ retracts rationally on its half-skeleton, by Proposition 6.5 there exists a connected CDGA, $Q$, of finite type such that $Q^{\geq n / 2-1}=0$ and $A_{P L}(\partial W) \simeq Q \oplus s s^{-n} \# Q$. Since $\partial W$ is 2-connected, 
we can assume that $Q$ is 2-connected. Then there exists a 1-connected CDGA model $R$ of $A_{P L}(W)$ and a surjective morphism

$$
\psi: R \rightarrow Q \oplus s s^{-n} \# Q
$$

that is a model of $A_{P L}(W) \rightarrow A_{P L}(\partial W)$.

Consider the following pullback diagram in CDGA

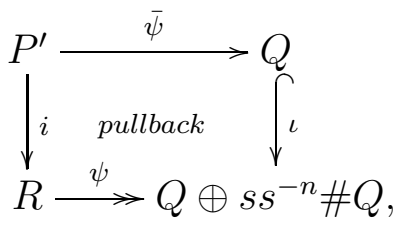

where $\iota$ is the obvious inclusion. This pullback is a homotopy pullback because $\psi$ is surjective.

We first prove that $P^{\prime}$ satisfies Poincaré duality in cohomology. We say that a CDGA morphism $\alpha: A \rightarrow \partial A$ satisfies relative Poincaré duality in cohomology in dimension $n$, if there exists an isomorphism of $\mathrm{H}(A)$-modules

$$
\mathrm{H}(\text { hoker } \alpha) \cong s^{-n} \# \mathrm{H}(A) .
$$

The morphism $\psi$ satisfies relative Poincaré duality because it is a model of the inclusion $\partial W \hookrightarrow W$. Moreover hoker $(\iota)$ is weakly equivalent to $s^{-n} \# Q$ as a $Q$-dgmodule, hence $\iota$ also satisfies relative Poincaré duality in dimension $n$. Since $\psi$ and $\iota$ satisfy relative Poincaré duality in degree $n$, a similar argument than the one used in [10, Theorem 2.1] implies that $\mathrm{H}\left(P^{\prime}\right)$ is a Poincaré duality algebra in degree $n$.

By Proposition 5.4 we can factorize $\bar{\psi}$ in CDGA as follows

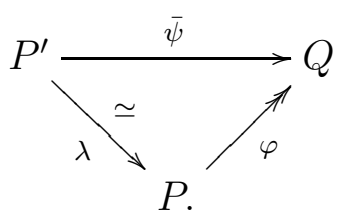

where $\varphi: P \rightarrow Q$ satisfies

(i) $P$ is a connected Poincaré duality CDGA in dimension $n$,

(ii) $\varphi$ is surjective, and

(iii) $Q^{\geq n / 2-1}=0$.

Since (11) is a homotopy pullback diagram, hoker $(i)$ is weakly equivalent as a $P^{\prime}$-dgmodule to hoker $(\iota) \simeq s^{-n} \# Q$. Therefore, there exists a cofibrant $P^{\prime}$-dgmodule $D$ with weak equivalences

$$
\text { hoker }(i) \underset{\gamma^{\prime}}{\stackrel{\simeq}{\simeq}} \underset{\gamma}{\simeq} s^{-n} \# Q \text {. }
$$

Set

$$
\varphi^{!}:=\theta_{P}^{-1} \circ s^{-n} \# \varphi: s^{-n} \# Q \rightarrow s^{-n} \# P \cong P,
$$

consider the natural map $l$ : hoker $(i) \rightarrow P^{\prime}$, and recall that $\lambda: P^{\prime} \rightarrow P$ is the quasi-isomorphism used to factorize the morphism $\psi$ through $P$. We can verify that $\mathrm{H}^{n}\left(\varphi^{!}\right)$is an isomorphism, hence $\varphi^{!}$is a shriek map 
or top degree map in the sense of [6, Section 5]. Also, the fact that $\mathrm{H}^{n}(R)=\mathrm{H}^{n}(W)=0$ implies that $\mathrm{H}^{n}(l)$ is also an isomorphism, and hence $\lambda \circ l$ is also a shriek map. Therefore, $\varphi^{!} \circ \gamma$ and $\lambda \circ l \circ \gamma^{\prime}$ are both shriek maps. The unicity of shriek maps up to multiplication by a scalar ([6, Proposition 5.6]) implies (multiplying $\gamma$ by a non-zero scalar if necessary), that the following diagram of $P^{\prime}$-dgmodules commutes up to homotopy

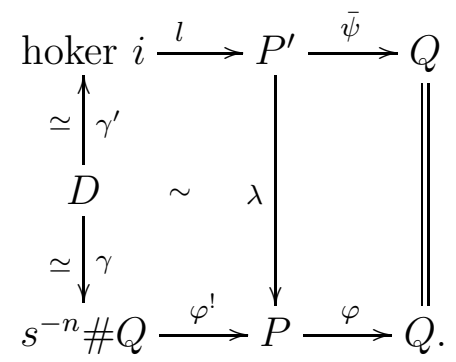

Composition induces a $P^{\prime}$-dgmodules morphism between the mapping cone of the morphism $l$ and the mapping cone of the morphism $\bar{\psi} \circ l$ :

$$
P^{\prime} \oplus_{l} s \text { hoker }(i) \rightarrow Q \oplus_{\bar{\psi} l} s \text { hoker }(i) .
$$

The homotopy commutative Diagram (12) implies that this last morphism is weakly equivalent as a $P^{\prime}$-dgmodules to the morphism

$$
P \oplus_{\varphi} ! s s^{-n} \# Q \rightarrow Q \oplus_{\varphi \varphi} ! s s^{-n} \# Q
$$

From Diagram (11) we get a quasi isomorphism $P^{\prime} \oplus_{l} s$ hoker $(i) \stackrel{\simeq}{\longrightarrow}$ $R$. Therefore, the morphism

$$
P^{\prime} \oplus_{l} s \text { hoker }(i) \rightarrow Q \oplus_{\bar{\psi} l} s \text { hoker }(i)
$$

is weakly equivalent to the morphism $\psi: R \rightarrow Q \oplus s s^{-n} \# Q$ which is a model of $A_{P L}(W) \rightarrow A_{P L}(\partial W)$. Standard techniques show that these morphisms are weakly equivalent as CDGA morphism. Since $\varphi \varphi^{!}=0$ for degree reasons, we deduce that the morphism

$$
\varphi \oplus i d: P \oplus_{\varphi} ! s s^{-n} \# Q \rightarrow Q \oplus s s^{-n} \# Q
$$

is a CDGA model of $\partial W \hookrightarrow W$.

Interestingly enough the hypothesis in Theorem 6.6 is only on the boundary $\partial W$. This implies that is $\partial W$ satisfies the hypothesis, then any other pair $\left(W^{\prime}, \partial W^{\prime}\right)$ with $\partial W^{\prime}=\partial W$ will also admits a pretty model. One can get many such manifolds $W^{\prime}$ by performing a connected sum in the interior of $W$ with a simply-connected closed $n$ manifold $N$. Or more generally we can modify $W$ by surgeries on its interior, which does not change its boundary. We can thus perform such surgeries on the manifolds from Example 6.4 to get many other examples. 


\section{AN OPEN QUESTION}

We finish this article by asking whether every Poincaré duality pair admits a pretty model. This would implies that every boundary manifold $\partial W$ admits a model of the form $Q \oplus_{\varphi \varphi} ! s s^{-n} \# Q$, which is a very special form of a Poincaré duality CDGA. Thus a preliminary algebraic question might be the following

Question 7.1. Let $(A, d)$ be a CDGA whose homology satisfies Poincaré duality in dimension $n-1$ and whose signature is 0 . Does it always exist a $C D G A, Q$, and a balanced $Q$-dgmodule morphism

$$
\Psi: s^{-n} \# Q \longrightarrow Q
$$

such that $(A, d)$ is quasi-isomorphic to

$$
Q \oplus_{\Psi} s s^{-n} \# Q \quad ?
$$

A positive answer to this question would be an interesting reinforcement of the main result of [7]. Note that we cannot drop the hypothesis of having zero signature since this is clearly the case for the CDGA $Q \oplus_{\Psi} s s^{-n} \# Q$. Of course a boundary manifold as $\partial W$ is always of zero signature. Conversely any rational Poincaré duality space with signature 0 appears as the boundary in some Poincaré duality pair, and hence if every Poincaré duality pair admits a pretty model then the answer to Question 7.1 will be affirmative.

\section{REFERENCES}

1. Hector Cordova Bulens, Pascal Lambrechts, and Don Stanley, Dgmodule models of configurations spaces in a manifold with boundary, in preparation.

2. with boundary, preprint.

3. Yves Félix, Stephen Halperin, and Jean-Claude Thomas, Rational homotopy theory, Graduate Texts in Mathematics, vol. 205, Springer-Verlag, New York, 2001. MR MR1802847 (2002d:55014)

4. Yves Félix and Jean-Claude Thomas, Rational BV-algebra in string topology, Bull. Soc. Math. France 136 (2008), no. 2, 311-327. MR 2415345 (2009c:55015)

5. Pascal Lambrechts and Don Stanley, The rational homotopy type of configuration spaces of two points, Ann. Inst. Fourier (Grenoble) 54 (2004), no. 4, 1029-1052. MR MR2111020 (2005i:55016)

6. _ Algebraic models of Poincaré embeddings, Algebr. Geom. Topol. 5 (2005), 135-182 (electronic). MR MR2135550 (2006g:55012)

7. Sci. Éc. Norm. Supér. (4) 41 (2008), no. 4, 495-509. MR 2489632 (2009k:55022)

8. _ A remarkable DGmodule model for configuration spaces, Algebr. Geom. Topol. 8 (2008), no. 2, 1191-1222. MR 2443112 (2009g:55011)

9. Dennis Sullivan, Infinitesimal computations in topology, Inst. Hautes Études Sci. Publ. Math. 47 (1977), 269-331 (1978). MR MR0646078 (58 \#31119)

10. C. T. C. Wall, Poincaré complexes. I, Ann. of Math. (2) 86 (1967), 213-245. MR 0217791 (36 \#880) 
18HECTOR CORDOVA BULENS, PASCAL LAMBRECHTS, AND DON STANLEY

H.C.B. And P.L.: IRMP, Université Catholique de Louvain, 2 Chemin du Cyclotron, B-1348 Louvain-la-Neuve, Belgium

E-mail address: hector.cordova@uclouvain.be

E-mail address: pascal.lambrechts@uclouvain.be

D.S.: University of Regina, Department of Mathematics, College West, Regina, CANADA

E-mail address: stanley@math.uregina.ca 